\title{
Aplicación de Técnicas de Validación de un Modelo de Simulación de Dinámica de Sistemas Caso de Estudio
}

\author{
Lisaura Walkiria Rodríguez Alvarado, Miguel Ángel López Ontiveros \\ Departamento de Ciencias Básicas e Ingeniería \\ Universidad Autónoma Metropolitana (UAM) \\ Ciudad de México, México \\ \{1wra,mlopez\}@correo.azc.uam.mx
}

\begin{abstract}
Resumen-La validación de un modelo constituye un paso fundamental para garantizar la confiabilidad de la información. En este artículo se presenta una aplicación de las técnicas de validación propuesta por varios autores para un modelo dinámico en un caso de estudio real de aplicación industrial, en el ramo del área metal-mecánica. Se presenta un esquema metodológico de varios aspectos y pasos de la validación formal del modelo propuesto, en el cual se especifica las pruebas de validación en dos etapas: estructura del modelo y estructura del comportamiento. Los principales resultados muestras que estas técnicas de validación disminuyen el riesgo asociado a errores potenciales del desarrollo del modelo y su representación con la realidad al ser comparado con eventos de la vida real
\end{abstract}

Palabras claves-Simulación de sistemas, modelado de sistemas continuos, verificación y validación.

\section{INTRODUCCIÓN}

El proceso de validación de un modelo consiste en determinar el grado en el cual un modelo corresponde a la adecuada representación del mundo real. Dentro del contexto de un modelo de Dinámica de Sistemas (DS) existen diferentes criterios de validación orientados a la estructura y al comportamiento, los cuales se pueden resumir en tres principales categorías de validación [1], [2]: Prueba estructural, pruebas orientadas al comportamiento y pruebas de patrones de comportamiento (políticas).

Dimitrios, Patroklos, y Eleftherios [3] establecen que, el principal criterio de validación de un modelo dinámico es la estructural, porque en esta se validan las relaciones usadas en el modelo. Esta opinión coincide con el planteamiento de Barlas [1] donde establece que el principio más importante de la validación de un modelo de DS es establecer la validación de la estructura del modelo.

Dimitrios, Patroklos, y Eleftherios, determinan que la validación de la estructura puede ser analizada mediante ciertos procedimientos y pruebas que se pueden clasificar en pruebas directas e indirectas. Ellos mencionan que el tipo de prueba directo es un método más estructurado de validación, siendo los más importantes, las pruebas de condición extrema y prueba de comportamiento de sensibilidad.

La revisión bibliográfica realizada en este artículo, sustenta el criterio establecido por estos autores, ya que se encontró que en la mayoría de los casos, la validación de los modelos desarrollados es verificada por el método de prueba directo.

La consistencia dimensional del modelo y el sometimiento del mismo a pruebas con condiciones extremas [4], es uno de los métodos usados en diferentes aplicaciones [5], [6], [7], [8] y [9] donde se evalúa cada ecuación del modelo y se conduce a una condición extrema, revisando si el modelo se comporta realísticamente bajo ciertas condiciones extremas.

Por otro lado, las pruebas de validación orientadas al comportamiento del modelo [1], originalmente sugerida por Forrester \& Senge [2], en las cuales se identifican los cambios en el comportamiento dinámico resultado del cambio en ciertos valores de los parámetros claves, es otra de las pruebas aplicadas.

Poles [10], identificó en su aplicación de validación con este tipo de pruebas, que los cambios en los patrones de comportamiento dinámico del modelo resultante de los cambios en los valores de los parámetros particulares corresponden con bastante precisión a los conocimientos disponibles de los sistemas del mundo real.

En el trabajo desarrollado por Qudrat-Ullah \& Seo Seong [11] validan el modelo de análisis de políticas energéticas con seis pruebas orientadas a la estructura y una prueba orientada al comportamiento, concluyendo que, aunque las pruebas de validez estructurales constituyen uno de los dos tipos generales de las pruebas necesarias para construir la confianza en un modelo de simulación de dinámica de sistemas, estas pruebas, son el núcleo del proceso de validación de modelos dinámicos y tienen precedencia temporal en pruebas de validez de comportamiento.

Towill [12], establece que, el paso final en la verificación de un modelo, debe ser la exposición pública del modelo ante la presencia de varios involucrados, principalmente ante los que han contribuido con la información y/o opiniones. Este criterio puede ser validado desde el punto de vista de Forrester, ya que menciona que un modelo dinámico es validado si es una adecuada representación del "modelo mental", el cual el modelador ha formado acerca del sistema. En su modelo de la empresa Sprague, Forrester dice que, fue empíricamente validado porque este crea un patrón de la misma naturaleza cualitativa como el sistema real.

Por otro lado, las opiniones de validación de estos modelos dinámicos con la aplicación de técnicas estadísticas son diversas, debido a que los datos generados en un modelo dinámico son en su mayoría autorrelacionados y correlacionados. Para aplicar pruebas estadísticas a datos auto/correlacionados, se requiere una extensiva simplificación del modelo o transformación de datos. Por otro lado, en este tipo de modelos no hay una única variable de salida, en la cual se puede enfocar el análisis estadístico para validar el modelo.

En el trabajo realizado por Martínez \& Richardson [13] exploran diferentes opiniones sobre las mejores prácticas de 
modelado, elegido de un distinguido grupo de expertos en el campo. Dentro de su evaluación, resaltan que el proceso de pruebas de modelo y evaluación en el proceso de modelado de dinámica de sistema, es descrita en la literatura principalmente por Randers [14], Richardson Pugh [15] y Sterman [4], aunque también no se puede omitir el enfoque de Barlas [1] y Forrester y Senge [2].

Martínez y Richardson presentan cuales son las prácticas más importantes del trabajo de modelado en el área de pruebas y evaluación del modelo. Como resultado del estudio, obtuvieron que, el aspecto de mayor importancia en las técnicas de validación es asegurar que el modelo sea consistente con datos históricos o con referencias del modo de comportamiento. Determinan como aspecto importante considerar la evaluación de los parámetros y análisis de resultados inesperados, y en mediana importancia, considerar al cliente final como involucrado en la evaluación, determinar los parámetros relevantes y su relación con el comportamiento del modelo.

Ellos concluyen que la mejor práctica identificada por el panel de expertos, es que se debe comparar los parámetros de comportamiento del modelo con datos reales, usando medidas estadísticas.

Esta opinión es compartida por Kleijnen [16], quien establece que desviaciones sistemáticas entre estadísticas observadas y valores teóricos pueden ser detectados a través de pruebas de distribución o pruebas paramétricas. Como ejemplo de esta apreciación se presenta el trabajo realizado por Chan \& Ang [17] en el cual compara el nivel de significancia entre los valores simulados y el nivel de inventario actual con ayuda de la herramienta estadística $\mathrm{T}$ student, la prueba fue desarrollada con variables significativas del modelo.

Por otro lado, Kleijnen [16] establece que la elección de la técnica estadística de validación depende de la disponibilidad de datos. Al no contar con datos reales para su análisis, la validación debe ser guiada por la teoría estadística de diseño de experimentos. Al contar únicamente con datos de salida, pero no de entrada la validación puede ser comparada con la técnica estadística T-Student. Y como tercer caso, al contar con información de entrada y salida se procede con técnicas de regresión y procedimientos de comparación.

En el trabajo presentado por Barlas [18], propone algunas de las medidas de estadística estándar (técnicas básicas de varianza, media y correlación) que se pueden utilizar para realizar la validación del modelo. El planteamiento de Barlas es utilizado en el trabajo desarrollado por Suryani, Chou, Hartono y Chen [19], en el que verifican la validación del modelo desarrollado mediante el análisis de la tasa de error generada por el promedio de datos históricos y el promedio de datos generados de la simulación.

El conjunto de métodos y enfoque de validación del modelo desarrollado en este artículo, se ha realizado siguiendo el camino propuesto por Forrester, Senge y Barlas, dado que no existe un método universal para validar un modelo dinámico, ya que está más vinculado con el objetivo del mismo.

El modelo dinámico de una línea de estampados de autopartes desarrollado por Rodríguez [20] se utilizará para ejemplificar la aplicación de las técnicas de validación bajo la metodología mencionada. Como primer paso, se propone validar la estructura del modelo, es decir que inicialmente se realizará la verificación estructural. Una vez que se haya verificado la arquitectura estructural se procede a utilizar la técnica estadística del valor absoluto de la media del error para complementar la validación del comportamiento del modelo comparando datos reales del sistema con los datos obtenidos vía simulación.

\section{MODELO DINÁMICO DEL FLUJO DE PRODUCCIÓN DE LA LÍNEA DE ESTAMPADOS}

El modelo dinámico que se presenta como caso de estudio, fue realizado en la línea de estampados de una empresa de autopartes, ubicada en la zona industrial de la ciudad de México. Esta empresa se dedica a la fabricación de componentes automotrices de acero y sus aleaciones, para el mercado de refacciones y automóviles originales de diferentes marcas y modelos. La línea de estampados de esta empresa consta de 15 prensas, 8 de ellas trabajan de modo manual y 7 trabajan en forma progresiva.

\section{A. Problemática de la empresa}

El comportamiento del proceso de planeación, control y producción de la línea de estampados, muestra un escenario complejo y variable, debido a la mezcla de producción a realizar y de las características de los requerimientos de producción. La asignación de carga de trabajo en las diferentes prensas basado en el criterio de prioridad de entrega del producto final no es suficiente.

La baja comunicación entre áreas y el desfase informativo generado por la falta de información en tiempo real, generan planteamientos erróneos desde el inicio de la planeación.

El proceso de organización administrativo va estrechamente ligado con el de planeación, debido a que las problemáticas anteriores se repiten constantemente.

El flujo de información no se hace presente en el registro de datos importantes del proceso de producción, lo que no permite obtener un panorama claro de la situación actual.

Y por último se puede determinar que la problemática principal que se presenta en el área de control, es la afectación de los tiempos de demora y por cambio de herramental y material en el cumplimiento del programa de producción. No hay un debido registro ni retroalimentación entre las partes involucradas, esto genera que el proceso de planeación se haga con desconocimiento de las condiciones de la capacidad actual de la línea.

\section{B. Desarrollo del modelo dinámico}

El esquema general de la estructura del modelo a desarrollar consta de dos módulos. En la Figura 1 se presenta el primer módulo el cual se refiere al análisis de la producción de componentes en cada prensa, mientras que en la Figura 2 se observa la estructura del segundo módulo el cual corresponde a la producción del producto terminado, este considera el flujo de producción del módulo uno y de otras estaciones de trabajo.

Debido a que el modelo propuesto involucra una serie de variables interrelacionadas, derivadas de más de dos mil componentes producidos en 15 prensas diferentes, fue necesario desarrollar una base de datos interactiva para el pre procesamiento de la información, desarrollada con ayuda de macros en Microsoft Excel. Esta base representa la fuente de alimentación de datos utilizados en la simulación del modelo, construido en la plataforma del software Vensim Ple ${ }^{\circledR}$ versión estudiantil.

En el módulo 1 se analizan las variables claves que afectan el desempeño del flujo productivo de cada prensa involucrada en el proceso, mientras que en el segundo módulo se analizan las afectaciones acumuladas de cada prensa reflejada en el cumplimiento de entrega del producto terminado al cliente. 


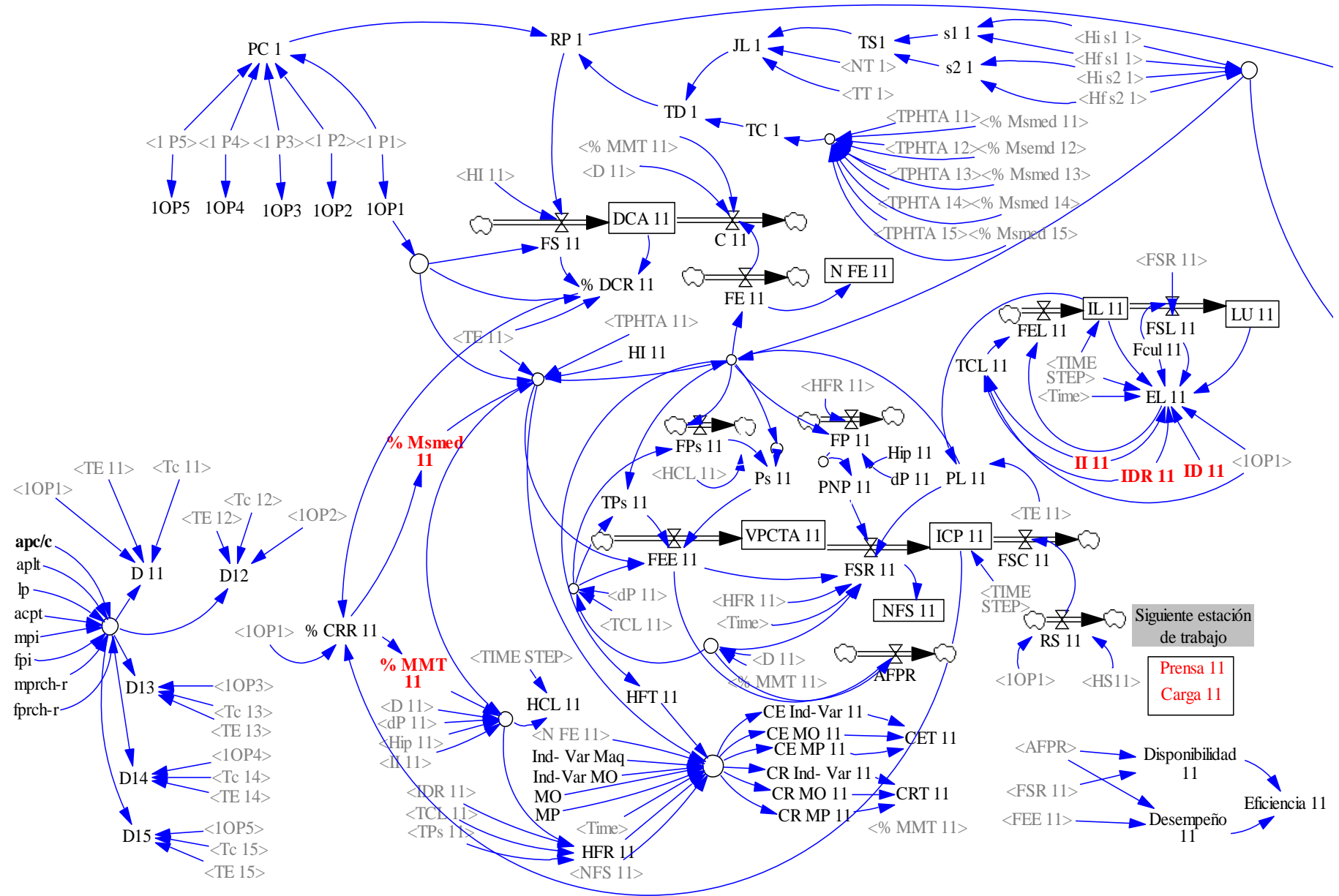

Fig. 1: Modelo dinámico módulo 1

\begin{tabular}{|c|c|c|c|c|c|c|c|c|}
\hline \multicolumn{9}{|c|}{ PRODUCTO TERMINADO } \\
\hline $\mathrm{PC} 1$ & $\mathrm{PC} 2$ & $\mathrm{PC} 3$ & $\mathrm{PC} 4$ & PC5 & P C6 & P C7 & $\mathrm{PC} 8$ & PRENSA \\
\hline $\mathrm{CCl}$ & $\mathrm{C} C 2$ & $\mathrm{CC} 3$ & $\mathrm{CC} 4$ & $\mathrm{CC} 5$ & C C6 & $\mathrm{CC} 7$ & $\mathrm{C}$ C8 & COMPONENTE \\
\hline$\overline{\mathrm{Std} C 1}$ & $\overline{\mathrm{Std} C 2}$ & $\overline{\mathrm{Std} C 3}$ & Std C4 & $\overline{\mathrm{Std} \text { C5 }}$ & Std C6 & Std C7 & Std C8 & ESTÁNDAR \\
\hline Cant C1 & Cant $\mathrm{C} 2$ & Cant C3 & Cant C4 & Cant C5 & Cant C6 & Cant C7 & Cant C8 & CANTIDAD \\
\hline $\begin{array}{l}\mathrm{HIC1} \\
\mathrm{C} / \mathrm{HC} 1\end{array}$ & $\mathrm{C} / \mathrm{HC} 2$ & $\mathrm{C} / \mathrm{HC} 3$ & $\mathrm{C} / \mathrm{HC} 4$ & $\mathrm{C} / \mathrm{HC} 5$ & $\mathrm{C} / \mathrm{HC} 6$ & $\mathrm{C} / \mathrm{HC} 7$ & $\mathrm{C} / \mathrm{HC} 8$ & COSTOS POR HORA \\
\hline
\end{tabular}

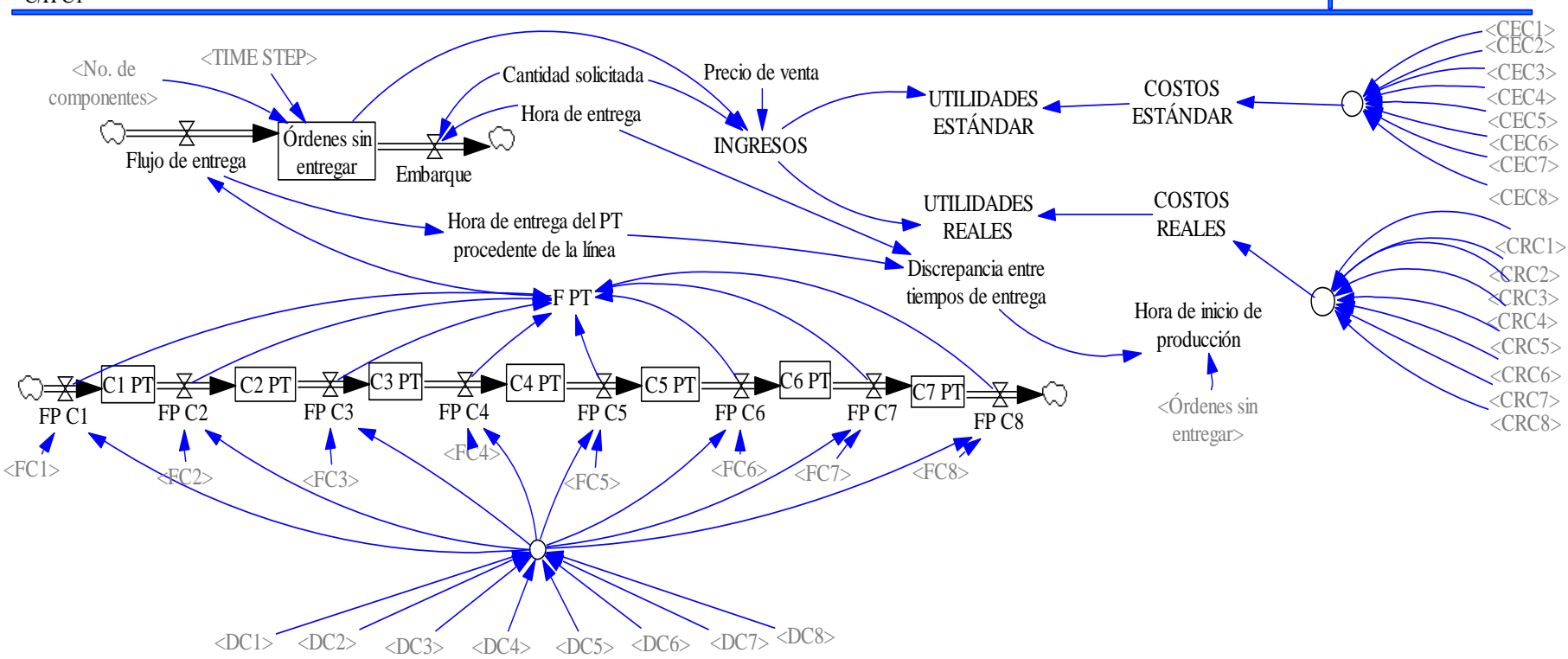

Fig. 2: Modelo dinámico módulo 2 
En este análisis se consideraron las siguientes variables principales para analizar y representar el comportamiento dinámico del proceso de administración de la producción de la línea de estampados.

- Producción de componentes generado de la diferencia de flujos de trabajo. Esta variable se refiere a la acumulación de la cantidad de componentes producidos obtenido de la diferencia de los flujos de entrada de producción normal (o esperada) y el flujo de producción afectado (por los diferentes paros y demoras). Esta variable representa un área de oportunidad del sistema analizado ya que al contabilizar la cantidad de componentes que se podrían producir en el tiempo representado por las afectaciones del flujo.

- Nivel de inventario de componente producido. Esta variable acumula la cantidad de componentes producidos en cada estación de trabajo, su valor está establecido por la diferencia entre el flujo de producción con afectaciones y el flujo de entrega de componentes a la siguiente estación.

- Porcentaje de capacidad real requerida. El porcentaje de capacidad requerida indica el porcentaje de mejora necesario para cumplir con las órdenes de producción en cada estación de trabajo. Está determinado por la diferencia de capacidad real y por el nivel de inventarios producidos. La diferencia de capacidad actual está establecida por la diferencia entre el ritmo con el que el cliente solicita los componentes y el ritmo con el cual la empresa puede dar respuesta a esta necesidad, considerado para cada estación de trabajo.

Por otro lado, la cantidad de inventario de componentes producidos indica si el lote de producción se ha entregado a tiempo y en la cantidad estimada por la orden de producción. De existir diferencias en relación a lo planeado, indica que hubo presencia de afectaciones en el flujo de producción, lo que se traduce en un incremento del porcentaje de capacidad requerido.

- Variación de costos reales y estimados. En cada estación de trabajo se contabilizan los costos directos e indirectos de mano de obra, maquinaria y material de acuerdo al comportamiento del flujo de producción normal y del flujo de producción con afectaciones. Esto permite visualizar un panorama comparativo entre estos dos estados.

- Porcentaje de contribución a la utilidad. El porcentaje de contribución a la utilidad se evidencia en el momento en que se hace efectivo el porcentaje de mejora basada en las dos propuestas planteadas. Esta variable se evalúa, de acuerdo al comportamiento de los costos e incremento del volumen de producción, resultante de considerar los porcentajes de mejora.

El conjunto de estas variables y su interrelación delimitan la frontera que explica el comportamiento de la línea de producción. Es importante esclarecer que el proceso a analizar inicia desde que se establecen las órdenes de producción y finaliza hasta que se obtiene el producto terminado, evaluando costos y porcentaje de contribución a la utilidad.

\section{VALIDACIÓN ESTRUCTURAL Y ESTADÍSTICA}

La validación es un proceso que sirve para crear confianza en el modelo y sus resultados. En primer lugar, se verificó la estructura del modelo, es decir, que se revisa su consistencia estructural. Como segundo paso se analizó el comportamiento del modelo utilizando la técnica estadística del valor absoluto de la media del error, donde se comparan los datos reales del

sistema con los datos obtenidos vía simulación. A continuación se explican los pasos considerados en este proceso.

\section{A. Análisis de la estructura del comportamiento}

Como primera fase de análisis se propone validar la arquitectura del modelo. Esto significa que se evalúa la estructura y parámetros del modelo directamente, sin excluir la relación entre estructura y comportamiento.

Este tipo de validación permite la identificación apropiada de la estructura, responsable del buen comportamiento del modelo en un proceso multidimensional es decir que incluye factores como: representación del problema, estructura lógica, relación causal y expresión matemática.

A continuación se presenta la aplicación de la prueba de validación estructural en el modelo desarrollado, la cual consta de 5 etapas.

- Prueba de Límites adecuados: La prueba estructural de límites adecuados considera la relación necesaria para satisfacer el propósito del modelo. Este tipo de prueba debe responder a la interrogativa si es necesario o no ampliar el modelo y si este incluye una estructura relevante.

Tomando en cuenta que el principal objetivo del modelo desarrollado es: analizar el comportamiento dinámico de la administración de un proceso de producción de bienes con enfoque a la contribución a las utilidades, en una industria metal-mecánica-línea de estampados, el modelo se ha constituido en dos etapas, realizado bajo el enfoque administrativo del proceso de producción.

El entorno en el cual se produce el comportamiento objeto de estudio está determinado desde que se establece el plan maestro de producción, lo cual se traduce en órdenes de producción de los diferentes componentes, hasta que se obtiene el producto terminado. Este procedimiento da paso a determinar las variables necesarias que se utilizan en el desarrollo del modelo, las cuales han sido procesadas con ayuda de macros en Microsoft Excel.

Para dar cumplimiento a la efectiva representación del objeto de estudio, contemplando todo su entorno, el modelo se ha constituido de dos etapas. Primeramente se analiza de manera independiente el proceso de producción de cada una de las prensas de la línea de estampados y posteriormente se integra en un segundo modelo correspondiente al flujo del producto terminado.

Para garantizar que el modelo desarrollo representa el comportamiento del sistema, este se ha hecho en conjunto con el personal involucrado en el proceso, quienes han brindado la retroalimentación necesaria para la construcción del mismo. El proceso de captura-registro y complementación de la información y la participación del conocimiento experto se obtuvo de dos fuentes de información. La primera es proporcionada directamente por la empresa y la segunda se obtuvo del registro, análisis, depuración y pre-procesamiento de la información obtenida en campo.

La información proporcionada por la empresa consiste en el programa de producción, programa de embarque, niveles de inventarios, características de los componentes a producir, costos directos e indirectos e información relacionada a la jornada laboral (turnos y horarios).Estos datos se obtuvieron directamente de registros, reportes y de bases de datos en el programa QAD (Software de planeación de recursos empresariales) que maneja la empresa.

Es importante mencionar que, actualmente este software tecnológico (QAD) no integra todos los sistemas del negocio lo que dificulta aún más la obtención de fuentes de 
información. La información recopilada en campo (en la línea de estampados), para su posterior registro, análisis, interpretación y pre-procesamiento, se refiere a las afectaciones que interrumpen el desempeño del flujo del proceso de producción. Estas afectaciones o fluctuaciones, como lo denominan algunos autores [23], se refieren a los tiempos de cambio de herramental y material y a las demoras ocasionadas por actividades anexas del proceso.

La obtención de esta información directamente de la línea, fue necesaria para garantizar la confiabilidad de la misma, ya que como se explicó anteriormente, no hay un debido registro de los tiempos de paros por cambio de herramental o material y demoras del proceso ni las afectaciones que le ocasionan. A continuación se presenta el análisis realizado para estas afectaciones.

- Verificación de la estructura. Verificar la estructura significa comparar la estructura directamente del modelo con la estructura del sistema real y modelos existentes, el cual no debe contradecir el conocimiento del mismo.

La verificación de la estructura del modelo desarrollado incluye una revisión exhaustiva de la literatura relevante relacionada al tema y se apoya en el conocimiento experto del sistema. Para garantizar un análisis crítico de la revisión literaria se realizó una primera selección de más de 150 publicaciones analizando el resumen, conclusiones y palabras claves. Posteriormente se realizó una revisión más detallada a un total de 70 publicaciones, esta selección estuvo orientada a las contribuciones, metodologías, métodos, aportaciones y enfoques de los diferentes investigadores.

Como resultado de la revisión bibliográfica se determinaron las perspectivas actuales de la aplicación de la DS en dos temáticas del campo de la manufactura: administración de la cadena de suministro y sistemas de producción. Por otro lado se examinaron las contribuciones académicas realizadas por diversos autores, las cuales se analizaron de acuerdo a su enfoque y aplicación.

La obra seminal de Jay Forrester, Industrial Dynamics ha sido la principal fuente de información estructural para el desarrollo del modelo, ya que representa el origen y las bases para el dominio de un modelo dinámico en materia de: simbología, estructura de relación causal y caracterización de variables.

En cuanto a estructura comparativa con modelos existentes, es evidente la aportación recibida de los modelos desarrollados por Deif y ElMaraghy [21]. Los cuales se han enfocado en el planteamiento y evaluación de políticas de capacidad dinámica enfocados a manufactura esbelta, y sistema de manufactura reconfigurable. Derivado de este trabajo se analiza la incorporación de indicadores claves en el modelo desarrollado del sistema y análisis de los efectos de tiempos de demora de ajustes en el sistema, así como el enfoque de costo analizado por etapas.

Es importante resaltar que el modelo desarrollado es un modelo personalizado adaptado a la necesidad de la situación real del sistema bajo estudio. Las aportaciones obtenidas de la revisión de la literatura sirvieron de base para plantear la estructura básica y analizar la integración de las diferentes variables interrelacionadas, evitando de esta manera omitir cualquier consideración que influya en el correcto funcionamiento del modelo.

- Consistencia Dimensional. La prueba de consistencia dimensional requiere que cada ecuación matemática en el modelo, así como la inclusión de parámetros sea evaluada y analizada .El entorno de simulación que ofrece el software Vensim Ple ® permite construir un modelo de forma amigable, que simulan los modelos en tiempo real mostrando su comportamiento. Este software añade facilidades para revisar la estructura de las ecuaciones del modelo y sus unidades de medida.

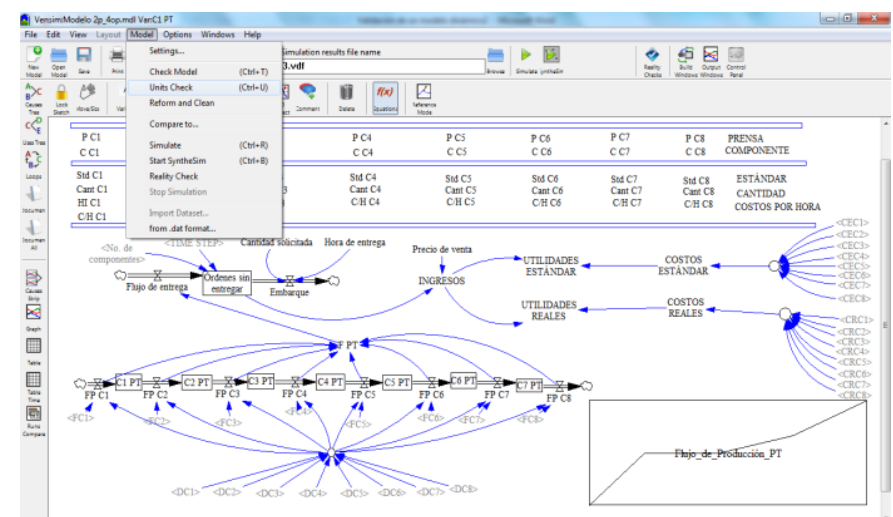

Fig. 3: Funciones en el software Vensim (Check Model y Units Check) para revisar sintaxis del modelo

En la Figura 3 se presenta la herramienta que ofrece el software Vensim Ple ${ }^{\circledR}$ para verificar el modelo. Por un lado dispone de una función para revisar la sintaxis del modelo (Check Model) y por otro lado una función para detectar errores en la coherencia de las unidades (Units Check). Esto permite que, a medida que se está realizando la construcción del modelo, y se vayan incorporando los diferentes parámetros, se realice una verificación paralela del modelo.

Considere el siguiente ejemplo, se verifica la consistencia de la ecuación del tiempo total de demoras (DPij) para la prensa 1 y orden de producción 1, con las herramientas disponibles en el software Vensim Ple ${ }^{\circledR}$. En ecuación 1 se presentan los parámetros y funciones de condicionalidad, donde el primer término de la ecuación indica los tiempos de demoras ocasionadas que dependen de la cantidad que se va a producir, mientras que el segundo y tercer término indican los tiempos de demoras ocasionadas de acuerdo a la frecuencia de ocurrencia en el tiempo de producción.

$$
\begin{aligned}
D P i j= & i O P j *\left(i f T c i j=1, \frac{\frac{a c c}{c}}{200}, \frac{a c p t+\frac{a c c}{c}+a c l t+l c}{200}\right) \\
& +\frac{i O P j}{T E i j}(\text { fpins } * \text { mpins })+\frac{i O P j}{T E i j}(\text { fprch_r } * \text { mprch_r })
\end{aligned}
$$

Los tiempos de demoras del proceso dependen de la cantidad de actividades anexas del proceso que se tengan que realizar durante el proceso de producción. Estas demoras dependen del tipo de componente a producir (1 o 2), el cual puede ser de dos tipos: manual o semiautomático. Los componentes de tipo manual presentan mayor tiempo de demoras que los componentes que se producen de modo semiautomático, ya que requiere de mayor cantidad de actividades anexas para desarrollar el proceso. En la Tabla I se presentan los parámetros utilizados en ecuación 1

En el recuadro rojo "1" de la Figura 4, se observa el estado de la ecuación antes mencionada. La sintaxis "Equation OK" indica que la ecuación es correcta, lo que establece que la ecuación ha sido desarrollada de forma idónea y apegada a los objetivos del modelo.

Verificación de Parámetros. Los parámetros deben ser verificados con el objetivo de analizar su correspondencia conceptual y numérica con las observaciones de la vida real, al igual que la estructura del modelo puede ser comparada con el conocimiento disponible. Los valores asignados a los parámetros del modelo desarrollado se derivan de dos fuentes, 
una proporcionada directamente por la empresa y otra información que fue necesaria procesar para obtener confiabilidad en la misma. En la Tabla II se enlistan las fuentes de obtención de los diferentes parámetros del modelo.

TABLA 1. PARÁmETROS UTILIZADOS EN ECUACIÓN 1

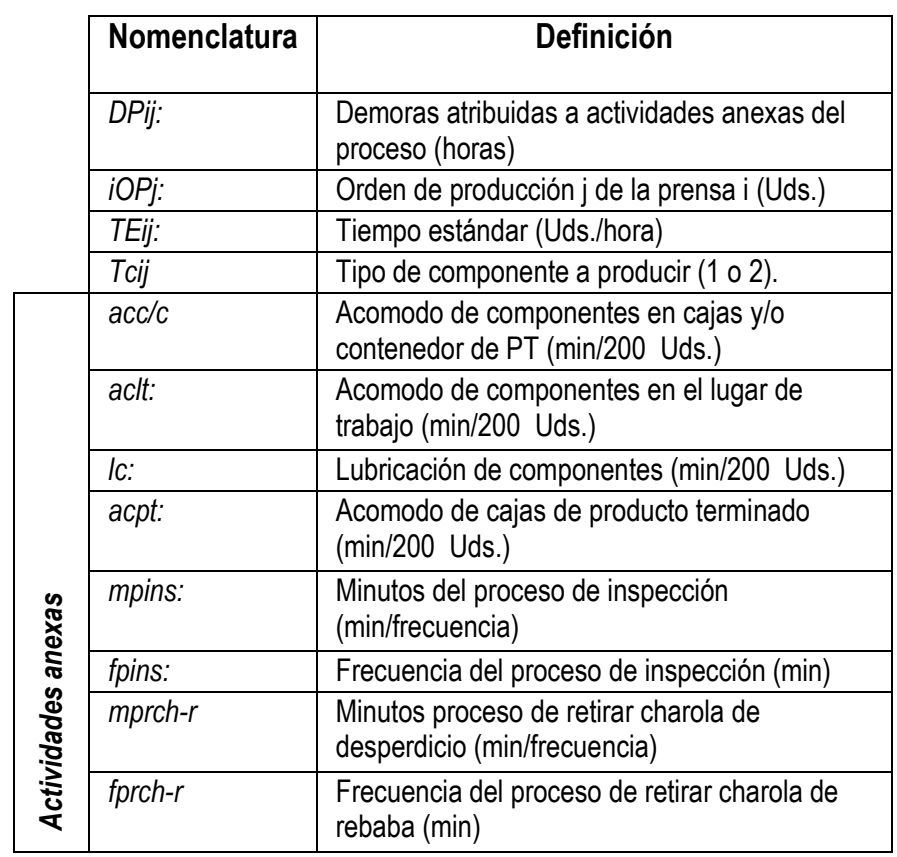

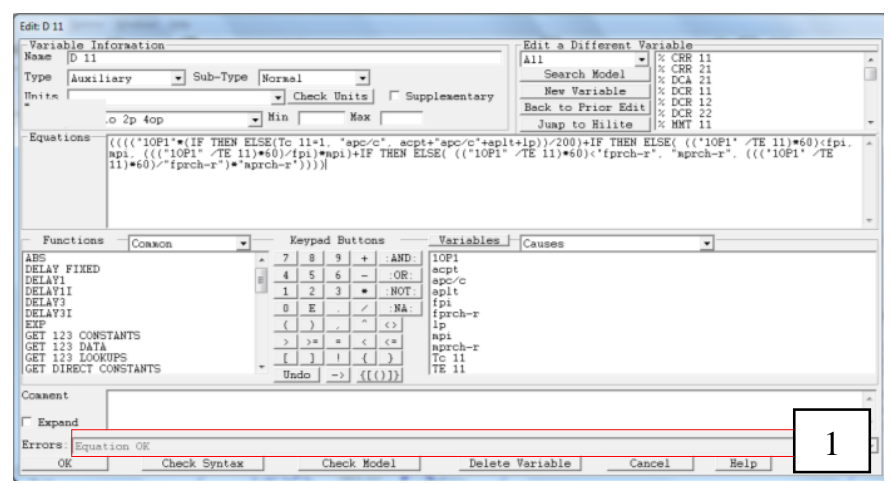

Fig. 4: Estado de la ecuación de tiempo total de demoras DPij

La información que proporciona la empresa corresponde al plan maestro de producción y del plan de producción de cada estación de trabajo. Estos datos son facilitados en el momento de realizar la simulación, los cuales varían de acuerdo a los días que se desean simular. Por otro lado, hay valores constantes como los datos de suplementos y datos de los costos de mano de obra e indirectos, los cuales son parámetros que no varían, y se incorporan una única vez al modelo.

Y por otro lado, están los datos que se obtuvieron de la captura, registro y pre-procesamiento de información del proceso, mediante la aplicación de la técnica de estudios de tiempos y movimientos así como técnicas de agrupación. Para garantizar la confiabilidad del procesamiento de los datos se realizaron pruebas estadísticas con la distribución Normal y Weibull, el porcentaje del error de la distribución que mejor se ajusta al pronóstico de los datos, se analizó mediante la desviación del valor absoluto de la media del error.

Pruebas de condiciones extremas. Un modelo que sólo se comporta aceptablemente bajo determinadas condiciones controladas, sólo puede ser útil para analizar políticas que hagan que el sistema actúe dentro de dichos límites. Por lo tanto, incorporar al modelo el conocimiento sobre condiciones

extremas mejora la comprensión y utilidad del mismo y por ende, su confianza. La estructura en el modelo de dinámica de sistemas debe permitir combinaciones extremas, permitiéndose ser cuestionado en relación a esto.

TABLA II. FUENTE DE OBTENCIÓN DE DATOS QUE ALIMENTAN EL MODELO PROPUESTO

\begin{tabular}{|l|}
\hline $\begin{array}{c}\text { Datos que alimentan el } \\
\text { modelo }\end{array}$ \\
\hline Porcentaje de mejora, \\
derivado de las \\
propuestas realizadas, \\
tiempo de cambio de \\
herramental y demoras \\
propias del proceso.
\end{tabular}

\section{Secuencia de} asignación de cargas para cada una de las prensas involucradas en el proceso a analizar
Órdenes de producción, tiempo estándar, turnos de la jornada laboral, tiempos suplementos, hora de entrega del producto terminado, costos de mano de obra y material

Fuente de obtención
Registro y análisis de la información
desarrollado. Para la obtención de los
tiempos promedios se efectuaron 45
observaciones de cada tipo de cambio de
herramental y material, cronometrando el
tiempo invertido por los operarios para
realizar el proceso. Una vez completado el
registro se calculó el tiempo promedio
obtenido y se calculó la desviación estándar
que presentan los datos respecto al tiempo
promedio real.
Nota: Se consideró este tamaño de muestra ya que se
considera que el comportamiento de los tiempos de
ejecución de una actividad sigue una distribución
normal, basado en esta consideración, es aceptable una
muestra superior a 30 observaciones.
Base de datos interactiva desarrollada en
Excel. Esta base cumple dos funciones: la
primera es que permite el fácil acceso de
los datos que alimentan el modelo
propuesto, ya que en esta base se agrupan
las variables necesarias para evaluar el
comportamiento del sistema. Como
segunda finalidad, brinda un escenario de
asignación de cargas de trabajo de cada
una de las prensas involucradas en el
proceso de producción.
Datos facilitados por la empresa, derivados
en su mayoría del programa de producción
y del plan de embarque.

Para la realización de las pruebas de condición extrema, Forrester y Senge sugieren que se deben examinar las ecuaciones de flujos, rastreada a través de ecuaciones auxiliares a los niveles establecidos en el modelo. Tomando en cuenta esta recomendación, los escenarios planteados para las pruebas de validez en condiciones extremas del modelo desarrollado, muestran un esquema comparativo de tres puntos clave del modelo: tiempos de demoras, paros no programados y falta de material. Se consideran puntos o situaciones claves ya que determinan las afectaciones en el flujo de producción lo que impacta en el cumplimiento de los tiempos de entrega de las órdenes de producción y a la vez en las utilidades.

Los ensayos bajo condiciones extremas de estos tres puntos clave se han desarrollado con valores extremos de cero e infinito (al considerar el valor de infinito es contemplar un valor muy largo aproximadamente un $300 \%$ de incremento sobre el valor original). Con el objetivo de ejemplificar los diferentes escenarios de evaluación de las pruebas de condición extrema, se ha considerado plantear el proceso de planeación del producto terminado (PT) CM-3910. Este producto involucra dos tipos de prensas de la línea de estampados, las cuales, a su vez cuentan con su propio escenario de cargas. 
Los valores de los parámetros obtenidos de la base interactiva desarrollada en Microsoft Excel, para analizar la prueba de condición extrema se muestran en la Tabla III. Estos corresponden a los diferentes componentes del producto terminado CM-3910. Los datos se han seleccionado para hacer una corrida de 1000 unidades.

TABLA III. DATOS DE LA CARGA DE PRODUCCIÓN DE PRENSA 1 Y 2

\begin{tabular}{|l|c|c|c|c|}
\cline { 2 - 4 } \multicolumn{1}{c|}{} & \multicolumn{3}{c|}{ Prensa 1/Prensa 2 } & \multirow{2}{*}{$\begin{array}{c}\text { Unidad de } \\
\text { Medida }\end{array}$} \\
\cline { 2 - 4 } \multicolumn{1}{c|}{$O P 1$} & $O P 2$ & $O P 3$ & Piezas \\
\hline Cantidad (CPC) & $2000 / 1200$ & $600 / 900$ & $1200 / 1000$ & Uds./hora \\
\hline $\begin{array}{l}\text { Estándar de } \\
\text { producción (STD } \\
\text { C) }\end{array}$ & $500 / 215$ & $150 / 120$ & $230 / 240$ & Min \\
\hline $\begin{array}{l}\text { Paro programado } \\
\text { (PPC) }\end{array}$ & $60 / 50$ & $30 / 40$ & $40 / 15$ & - \\
\hline $\begin{array}{l}\text { Tipo de cambio } \\
\text { (TC) }\end{array}$ & $1 / 1$ & $2 / 2$ & $2 / 2$ & Horas \\
\hline Hora de inicio & $7 / 7$ & - & - & \\
\hline
\end{tabular}

Escenario 1: Tiempos de demoras y su afectación sobre: capacidad de producción, cantidad de componentes que se podrían producir y costos reales de producción.

En la Figura 5 se aprecia que, al incurrir en un tiempo de demora igual a cero, el comportamiento de las variables es favorable, ya que al disminuir los tiempos de demora, no se registran afectaciones en el flujo y no es necesario un incremento en la capacidad real del sistema, por otro lado el comportamiento de los costos reales se ajusta al comportamiento del costo estándar, es decir que ocurre bajo lo pronosticado.

Este escenario contrasta en gran medida con el segundo escenario planteado (ver Figura 6), en el cual se incrementa sustancialmente los tiempos de cada una de las actividades anexas al proceso ocasionando que los tiempos de demoras incrementen aproximadamente un $1000 \%$ de su valor normal. Bajo condiciones normales, el tiempo de demora total que se generaría (en este caso) de las actividades anexas al proceso sería de 30 minutos aproximadamente, al incrementar 1000\% su valor, se está considerando un tiempo total de demora aproximadamente de 300 minutos.

En la Figura 6 es evidente el comportamiento de las variables ante el incremento del tiempo de demoras. Al incrementar el tiempo de demoras de 30 a 300 minutos. La cantidad de componentes dejados de producir asciende a 2100 componentes, lo cual tiene sentido si se compara con el estándar de producción de 500 piezas por hora. Esto se respalda al analizar el comportamiento del porcentaje de la capacidad requerida, donde indica que necesita un $100 \%$ para cumplir con la orden de producción. Finalmente los costos reales en relación al costo estándar presentan un incremento sustancial, pasando de 4600 a 7000 unidades monetarias, esto debido a que el tiempo empleado para cumplir la orden de producción se vio afectado por el incremento del tiempo de las demoras.

Escenario 2: Falta de material: incumplimiento en las órdenes de producción.

El dato de inventario disponible real lo que permite analizar es que, en cualquier momento el flujo del proceso puede ser interrumpido por falta de material, esto ocasionado por una mala comunicación entre las áreas de producción y control de inventario. Para el análisis de este escenario se plantea la situación de paro de flujo de producción indefinido por falta de lámina. En la Figura 7 se presenta el efecto que ocasiona la falta de material en el inventario de componentes Rodrígez Alvarado, L., López Ontiveros, M.. 2016. Aplicación de Técnicas de Validación de un Modelo de Simulación de Dinámica de Sistemas, Caso de Estudio producidos y en el cumplimiento de las órdenes de producto terminado.

(a) Cantidad de componentes posibles a producir

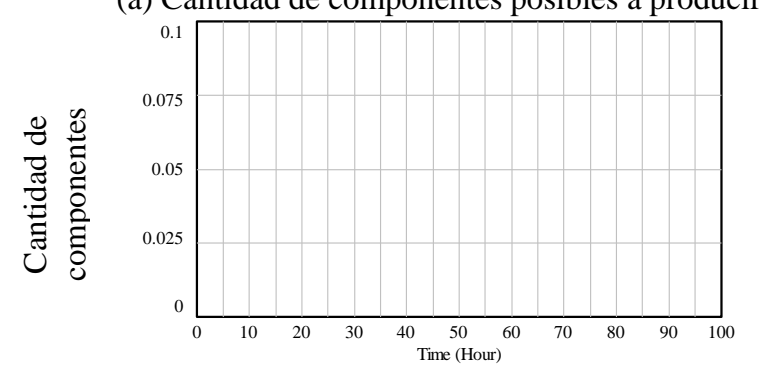

(b) Porcentaje de capacidad requerida real

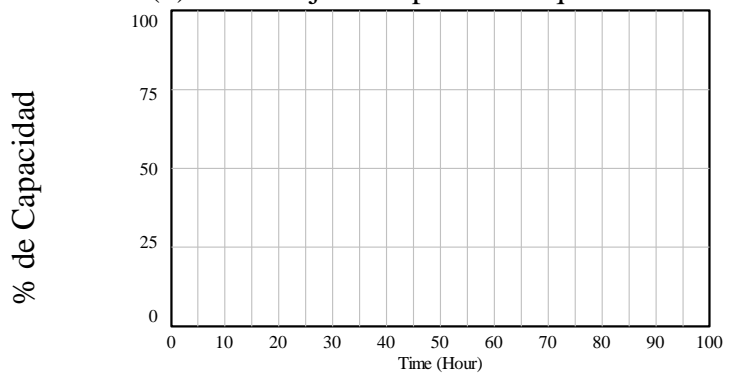

(c) Costo estándar total

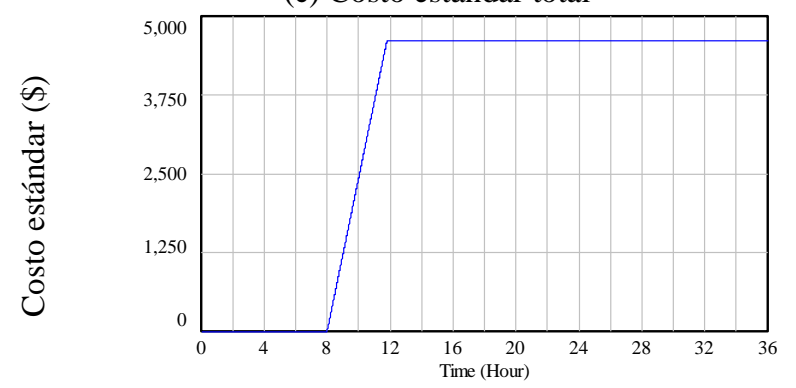

(d) Costo real total

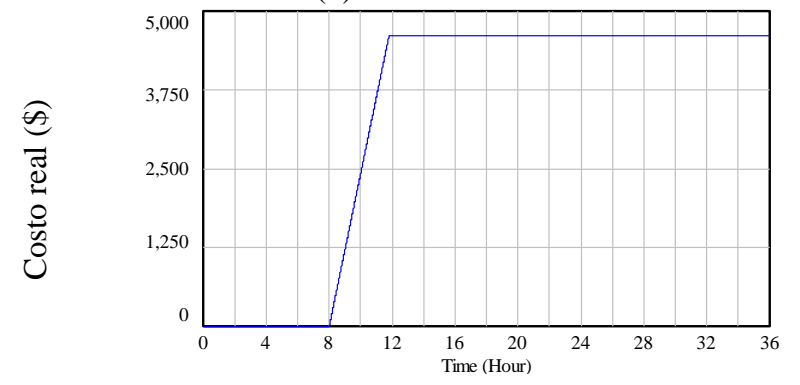

Fig. 5: Comportamiento de cantidad de piezas dejadas de producir (a), Porcentaje de capacidad requerida (b), Costo estándar total (c) y Costo real total (d)

Para comprender este escenario, es necesario plantear la situación completa. El inventario disponible indica que hay material suficiente para producir 2000 piezas más (1000 piezas más que las solicitadas en la orden de producción), es decir que el inventario de material es suficiente para completar la orden de producción.

Sin embargo, una vez iniciado el proceso de producción, se notifica que en el inventario disponible realmente hay 500 unidades, considerando que el inventario inicial es de 1000 unidades, lo que permite producir únicamente la cantidad de 1500 piezas. Este panorama se ve reflejado en la Figura 7-a, en la que el nivel de inventario de componentes producidos alcanza el nivel de 1500 piezas, en ese momento se hace la entrega de la cantidad producida, quedando un faltante de 500 piezas sin producir. 
(a) Cantidad de componentes posibles a producir

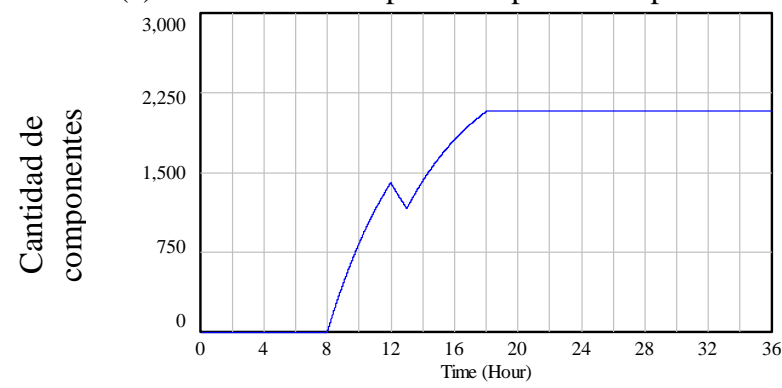

(b) Porcentaje de capacidad requerida real

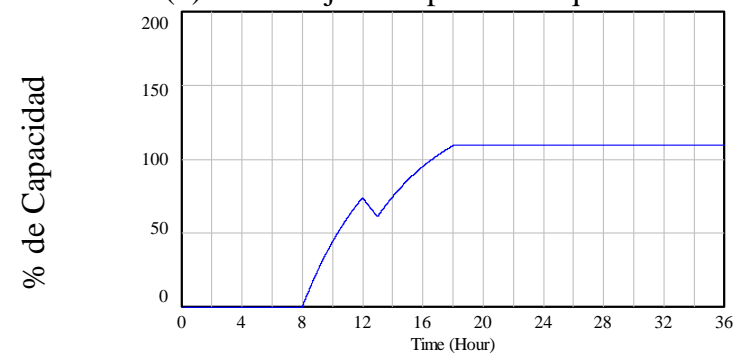

(c) Costo estándar total

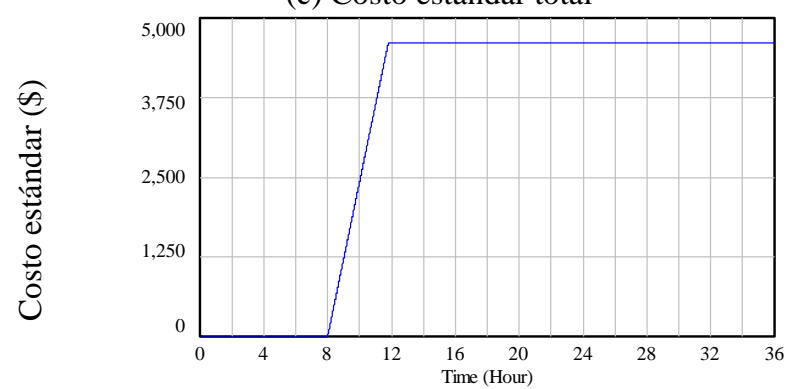

(d) Costo real total

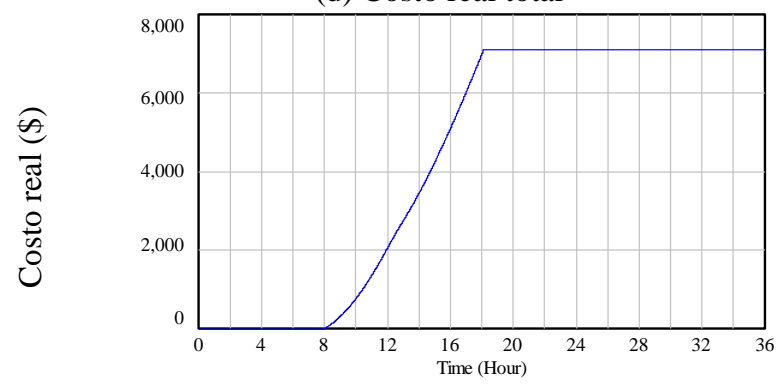

Fig. 6: Comportamiento de cantidad de piezas dejadas de producir (a), Porcentaje de capacidad requerida (b), Costo estándar total (c) y Costo real total (d)

Esta afectación se ve reflejada en la Figura 7-b, en el que se observa el comportamiento de unidades de producto terminado que se generan a lo largo del flujo de producción. Bajo este escenario, al finalizar del turno (tomando en cuenta las otras estaciones de trabajo y el flujo completo) se quedan sin entregar 1000 unidades de producto terminado.

Escenario 3: Paros no programados, con afectación en el cumplimiento del plan de producción de otras prensas.

Los tiempos de paros no programados presentan la característica que pueden aparecer en cualquier momento del flujo, ocasionando una pausa de tiempo determinado, hasta que el problema sea resuelto y el flujo sea reestablecido. Para el análisis de escenario de este factor, se tomó en cuenta un paro no programado de 5 horas.

(a) Inventario de componentes producidos

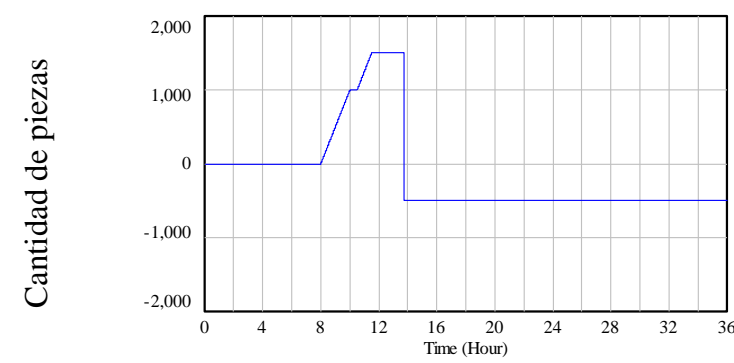

(b) Ordenes sin entregar

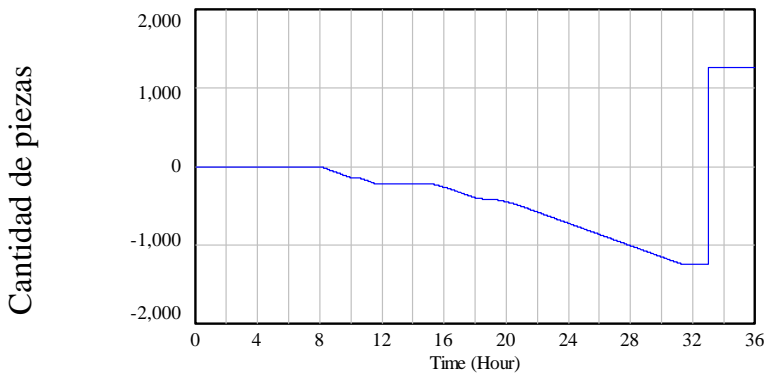

Fig. 7: Inventario de componentes producidos (a) y Cantidad de órdenes sin entregar (b)

En la Figura 8-a se observa que el tiempo de paro no programado de 5 horas afecta la producción, generando una producción retrasada aproximadamente 2700 piezas. Sin embargo este retraso no únicamente influye en el flujo de producción de esa prensa, sino que afecta el tiempo de entrega del componente a la siguiente prensa (relación entre módulo 1).

(a) Cantidad de componentes retrasados en la

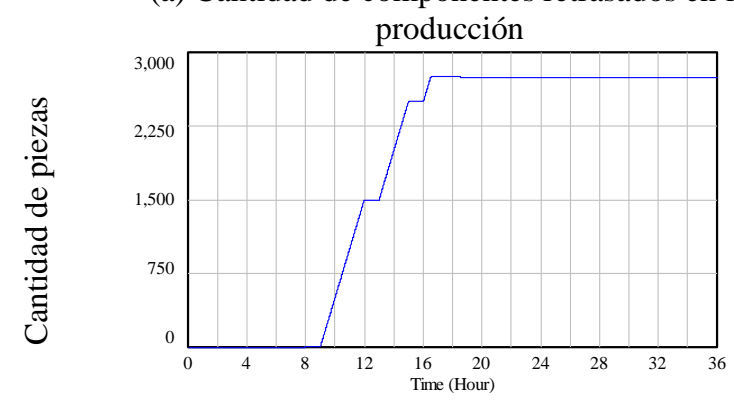

(b) Inventario de componentes producidos

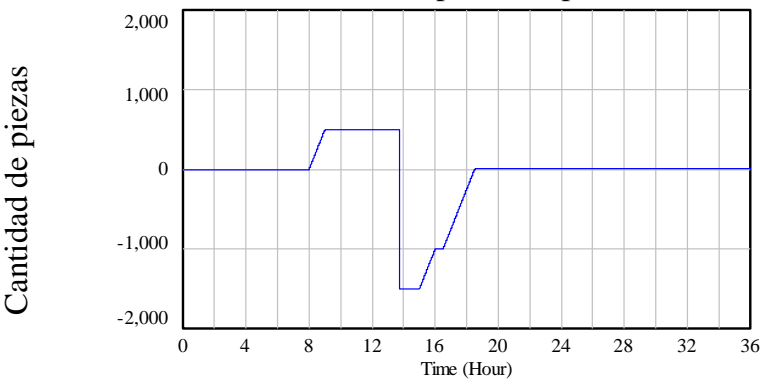

Figura 8: Cantidad de piezas dejadas de producir (a) e Inventario de componentes producidos (b)

En la Figura 8-b se observa que el inventario de componentes producidos asciende a 500 piezas en el intervalo del tiempo de las 8 a 12 horas. De acuerdo con el programa de producción, la entrega del lote de producción de 2000 piezas, a una velocidad de tiempo estándar de 500 piezas por hora debería haber sido 4 horas posteriores a su inicio de producción (entre la hora 12 y 13). Sin embargo para esa hora, hay un faltante de 1500 piezas, las cuales se entregan hasta las 18 horas aproximadamente. 
Validación estadística. Para el análisis de validación estadística se realizaron un total de 43 simulaciones ejecutadas comprendidas entre la semana 1 y la semana 43 del año 2014. Durante este periodo se evaluó el desempeño del modelo propuesto y se establecieron los datos comparativos resultantes de la simulación con un total de 5,925 datos registrados. La distribución de datos analizados se presenta en la Figura 9.

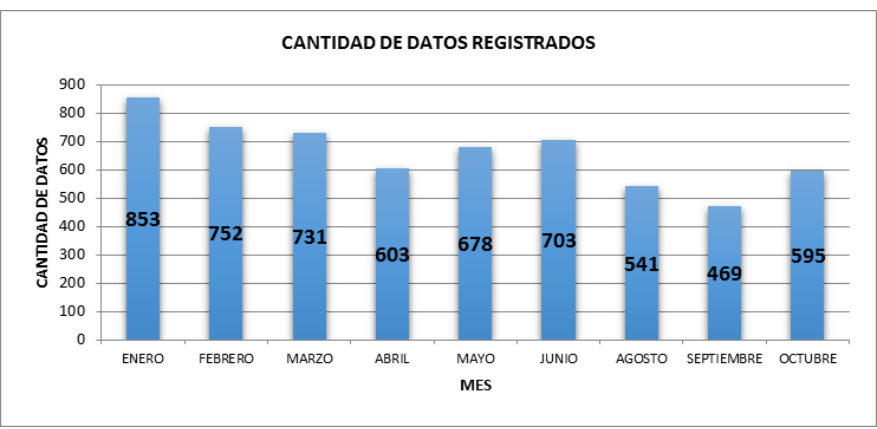

Fig. 8: Distribución de datos registrados durante el período de evaluación

Se utilizó la técnica estadística del error relativo para analizar el porcentaje de error generado de los datos reales y simulados. De acuerdo con Barlas [2] y su estudio realizado en relación a validación de modelos de simulación, establece que un modelo será válido si la tasa de error es más pequeña que el 5\%. Tomando en consideración, se utilizó el porcentaje de error relativo absoluto para determinar la confiabilidad del modelo. Su expresión matemática se establece en ecuación 2.

$$
\% \text { error relativo }=\left|\frac{\text { dato simulado }- \text { dato real }}{\text { dato real }}\right| * 100
$$

Las variables seleccionadas para realizar el análisis de medida el comportamiento del objeto de estudio. Para tal efecto se consideraron: Tiempo improductivo, generado de las afectaciones de los diferentes tiempos de paros y demoras, Tiempo de entrega entre estaciones y los indicadores de disponibilidad, desempeño y eficiencia.

En la Tabla IV se resume el promedio del comportamiento estadístico de los datos analizados. Del análisis de estos datos se determina que, la media obtenida entre los datos reales y datos obtenidos vía simulación presentan poca variación, lo que indica que son medias aproximadas. El porcentaje del valor absoluto de la media del error obtenido de cada una de representación del modelo propuesto es confiable.

TABLA IV. COMPORTAMIENTO ESTADÍSTICO PROMEDIO DE LOS DATOS REALES Y DATOS SIMULADOS

\begin{tabular}{|c|c|c|c|c|c|}
\hline & \multicolumn{5}{|c|}{ PROMEDIO GENERAL } \\
\hline & \begin{tabular}{|c|} 
Tiempo \\
improductivo \\
(min)
\end{tabular} & \begin{tabular}{|c|}
$\begin{array}{c}\text { Tiempo de } \\
\text { entrega } \\
(\mathrm{min})\end{array}$ \\
\end{tabular} & $\begin{array}{l}\text { Disponibilidad } \\
\text { (\%) }\end{array}$ & \begin{tabular}{|c|} 
Desempeño \\
$(\%)$
\end{tabular} & $\begin{array}{c}\text { Eficiencia } \\
\%\end{array}$ \\
\hline \multicolumn{6}{|l|}{ Datos reales } \\
\hline Media & 331.32 & 467.49 & $85.26 \%$ & $44.36 \%$ & $36.74 \%$ \\
\hline Desviación estándar & 331.08 & 360.08 & $15.40 \%$ & $23.28 \%$ & $20.08 \%$ \\
\hline Dato máximo & 1461.21 & 1563.33 & $100.00 \%$ & $99.52 \%$ & $92.36 \%$ \\
\hline Dato mínimo & 9.00 & 19.33 & $15.22 \%$ & $5.03 \%$ & $4.15 \%$ \\
\hline \multicolumn{6}{|l|}{ Datos simulados } \\
\hline Media & 335.25 & 471.42 & $85.22 \%$ & $44.09 \%$ & $36.48 \%$ \\
\hline Desviación estándar & 336.98 & 366.28 & $15.43 \%$ & $23.48 \%$ & $20.11 \%$ \\
\hline Dato máximo & 1552.04 & 1680.00 & $100.00 \%$ & $102.10 \%$ & $94.30 \%$ \\
\hline Dato mínimo & 7.44 & 19.10 & $14.46 \%$ & $5.26 \%$ & $5.10 \%$ \\
\hline$\%$ de error datos reales/simulados & $4.02 \%$ & $2.72 \%$ & $0.28 \%$ & $2.95 \%$ & $2.60 \%$ \\
\hline
\end{tabular}
validación son representativas del sistema y resumen en gran las variables analizadas es menor al $5 \%$, esto indica que la

La variable Tiempo improductivo presenta el porcentaje de error más elevado (4.02\%). Esto se le atribuye a la variabilidad que presentan los tiempos de demoras con relación a la cantidad y tipo de componente a producir. A diferencia del indicador de Disponibilidad, el cual presenta el porcentaje de error más bajo $(0.28 \%)$, debido a que esta variable depende directamente de los paros programados y del flujo esperado del proceso de producción.

\section{CONCLUSIONES}

A lo largo del documento se presenta el proceso para determinar el grado en el cual el modelo de simulación representa adecuadamente la realidad del proceso de ensamble, analizando el comportamiento continuo de los flujos de producción y el ajuste del proceso del sistema ante el efecto de eventos que ocasionan cambios en el plan original. Por otro lado, se evalúa el porcentaje en la contribución a la utilidad considerando un porcentaje de mejora en el tiempo de producción derivado de dos propuestas planteadas, SMED (single minute exchange of die) y MMT (mejora en el método del trabajo).

En la aplicación de las técnicas de validación se consideró el análisis de estructura y comportamiento en el que se demuestra que el modelo dinámico es capaz de reproducir el comportamiento observado de la línea de ensamble y responde positivamente a implicaciones esenciales como límites extremos.

Por lo tanto se puede afirmar que el modelo tiene la habilidad de representar la realidad de la situación actual de la línea, lo cual contribuye a los modeladores en el dominio de la comprensión del objetivo del modelo.

\section{REFERENCIAS}

[1] Barlas, Y., 1994. Model Validation in System Dynamics. The 12th International Conference of the System Dynamics Society (pp. 1-10). Scotland: Clare Monaghan and Eric Wolstenholme.

[2] Forrester, J., Senge, P., 1980. Tests for building confidence in system dynamics models. TIME Studies in the Management Science 14, 209-228.

[3] Dimitrios, V., Patroklos, G., \& Eleftherios, I., 2007. A system dynamics model for dynamic capacity planning of remanufacturing in closed-loop supply chains. Computers \& Operations Research 34, 367-394.

[4] Sterman, J., 2000. Business Dynamics: Systems Thinking and Modeling for a Complex World. New York: McGraw-Hill Higher Education.

[5] Debabrata, D., Pankaj, D., 2013. A system dynamics framework for integrated reverse supply chain with three way recovery and product exchange policy. Computers \& Industrial Engineering 60, 720-733.

[6] Patroklos, G., Alexandra, P., 2013. Dynamic Drum-Buffer-Rope approach for production planning and control in capacitated flow-shop manufacturing systems. Computers \& Industrial Engineering 65, 689-703.

[7] Patroklos, G., Charalampos, M., 2012. Real-time production planning and control system for job-shop manufacturing: A system dynamics analysis. European Journal of Operational Research 216, 94-104.

[8] Souza, G., 2004. Impact of Alternative Flow Control Policies on Value Stream Delivery Robustness Under Demand Instability: a System Dynamics Modeling and Simulation Approach. Blacksburg, VA: Faculty of the Virginia Polytechnic Institute.

[9] Peña Z., Crespo M., 2003. Dinámica de Sistemas y Análisis Cualitativo Matemático, en Modelos de Gestión de la Producción. Sevilla: Escuela de Ingenieros Industriales.

[10] Poles, R., 2013. System Dynamics modelling of a production and inventory system for remanufacturing to evaluate system improvement strategies. Int. J. Production Economics, 189-199.

[11] Qudrat-Ullah, H., Seo Seong, B., 2010. How to do structural validity of 
a system dynamics type simulation model: The case of an energy policy model. Energy Policy 38, 2216-2224.

[12] Towill, D., 1996. Industrial dynamics modelling of supply chains. Logistics Information Managment 9, 43-56.

[13] Martinez-Moyano, I., Richardson, G., 2013. Best practices in system dynamics modeling. System Dynamics Review 29, 102-123.

[14] Randers, J., 1980. Elements of the System Dynamics Method. Cambridge, MA.: Productivity Press.

[15] Richardson, G., Pugh, A., 1981. Introduction to System Dynamics Modeling with DYNAMO. Cambridge, MA: Productivity Press.

[16] Kleijnen, J., 1995. Verification and validation of simulation models. European Journal of Operational Research 82, 145-162.

[17] Chan, K., Ang, C.-L., 2011. Supply Chain Management: A System Dynamics Approach to Improve Visibility and Performance. Journal of Information and Communication Technology, 117-135.

[18] Barlas, Y. (1996). Formal aspects of model validity and validation in system dynamics. System Dynamics Review 12, 183-210.

[19] Suryani, E., Chou, S.-Y., Hartono, R., Chen, C.-H., 2010. Demand scenario analysis and planned capacity expansion: A system dynamics framework. Simulation Modelling Practice and Theory, 732-751.

[20] Rodriguez, L., 2015. Diseño de un Modelo Dinámico de Administración de la Producción de Bienes, con base en la Contribución a las Utilidades. México, Instituto Politécnico Nacional.Proyecto de tesis no publicado.

[21] Deif, A; ElMaraghy, H., 2009 Modelling and analysis of dynamic capacity complexity in multi-stage production. Production Planning \& Control Vol. 20, No. 8, December 2009, 737-749

[22] Barlas, Y. (1989). Multiple tests for validation of system dynamics: type of simulation models. European Journal of Operational Research 42 (1989) 59

[23] Everett E. Adam y Ronald J. Ebert. Administración de la producción y operaciones. 4ta edición. Prentice-Hall.1991.

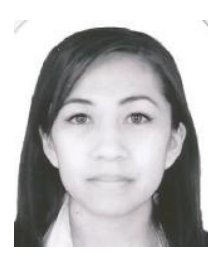

Lisaura Walkiria Rodríguez Alvarado es ingeniera industrial por la facultad de ingeniería Industrial de la Universidad Nacional de Ingeniería (UNI-Managua, Nicaragua). Estudió una Maestria en Ingeniería en Manufactura en el Instituto Politécnico Nacional de México y posteriormente estudió el doctorado en Ciencias en Ingeniería Mecánica en esta misma institución. Es docente de la carrera de ingeniería industrial de la Universidad Autónoma Metropolitana e investigador del área de innovación de sistemas de esta misma universidad. Sus áreas de interés son: modelado y simulación de sistemas productivos, mejora continua, comportamiento dinámico de sistemas.

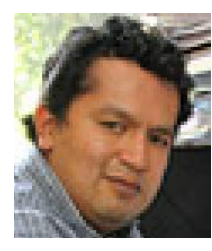

Miguel Ángel López Ontiveros es ingeniero industrial por el departamento de sistemas de la Universidad Autónoma Metropolitana (UAM). Estudió una Maestría en Ingeniería en Sistemas en el Instituto Politécnico Nacional y posteriormente realizó sus estudios de doctorado en Institut National Polytechnique de Grenoble -Francia. Es docente y coordinador de la carrera de ingeniería industrial de la Universidad Autónoma Metropolitana. Sus áreas de interés son: Diseño Integral, cadena de suministro, reingeniería y sistemas de producción. 